\title{
TU/e EnNHOUN

\section{Communications and sensing of illumination contributions in a power LED lighting system}

\section{Citation for published version (APA):}

Linnartz, J. P. M. G., Feri, L., Yang, H., Colak, S. B., \& Schenk, T. C. W. (2008). Communications and sensing of illumination contributions in a power LED lighting system. In IEEE International Conference on Communications, 2008 : ICC '08 ; 19 - 23 May 2008, Beijing, China (pp. 5396-5400). Institute of Electrical and Electronics Engineers. https://doi.org/10.1109/ICC.2008.1011

DOI:

10.1109/ICC.2008.1011

Document status and date:

Published: 01/01/2008

\section{Document Version:}

Publisher's PDF, also known as Version of Record (includes final page, issue and volume numbers)

\section{Please check the document version of this publication:}

- A submitted manuscript is the version of the article upon submission and before peer-review. There can be important differences between the submitted version and the official published version of record. People interested in the research are advised to contact the author for the final version of the publication, or visit the $\mathrm{DOI}$ to the publisher's website.

- The final author version and the galley proof are versions of the publication after peer review.

- The final published version features the final layout of the paper including the volume, issue and page numbers.

Link to publication

\section{General rights}

Copyright and moral rights for the publications made accessible in the public portal are retained by the authors and/or other copyright owners and it is a condition of accessing publications that users recognise and abide by the legal requirements associated with these rights.

- Users may download and print one copy of any publication from the public portal for the purpose of private study or research.

- You may not further distribute the material or use it for any profit-making activity or commercial gain

- You may freely distribute the URL identifying the publication in the public portal.

If the publication is distributed under the terms of Article 25fa of the Dutch Copyright Act, indicated by the "Taverne" license above, please follow below link for the End User Agreement:

www.tue.nl/taverne

Take down policy

If you believe that this document breaches copyright please contact us at:

openaccess@tue.nl

providing details and we will investigate your claim. 


\title{
Communications and Sensing of Illumination Contributions in a Power LED Lighting System
}

\author{
Jean-Paul M. G. Linnartz ${ }^{* \dagger}$, Lorenzo Feri*, Hongming Yang*†, Sel B. Colak* and Tim C. W. Schenk* \\ * Philips Research, High Tech Campus 37, Eindhoven, The Netherlands, j.p.linnartz@philips.com \\ $\dagger$ Eindhoven University of Technology, Eindhoven, The Netherlands
}

\begin{abstract}
In recent years, LED technology emerged as a prime candidate for the future illumination light source, due to high energy efficiency and long life time. In addition, LEDs offer a superior flexibility in terms of colors and shapes, which leads to a potentially infinite variety of available light patterns. In order to create these patterns via easy user interaction, we need to sense the local light contribution of each LED. This measurement could be enabled through tagging of the light of each LED with unique embedded IDs. To this end, we propose a new modulation and multiple access scheme, named as codetime division multiple access - pulse position modulation (CTDMA$P P M)$ : a form of PPM which is keyed according to a spreading sequence, and in which the duty cycle is subject to pulse width modulation (PWM) according to the required lighting setting. Our scheme considers illumination constraints in addition to the communication requirements and, to our best knowledge, it has not been addressed by other optical modulation methods. Based on the proposed modulation method and multiple access schemes, we develop a system structure, which includes illumination sources, a sensor receiver and a control system. Illumination sources illuminate the environment and transmit information, simultaneously. According to our theoretical analysis, this system structure could support a number of luminaries equal to the size of the CDMA codebook times the dimming range.
\end{abstract}

\section{INTRODUCTION}

Light emitting diodes (LEDs) will capture a significant portion of the market for illumination and may largely replace incandescent and gas discharge lamps in the future [1]. LED technology allows control over the light intensity, light color and illumination pattern. Hence, the concept of pure illumination may shift into dynamic lighting atmosphere provisioning. It is recognized that smart, intuitive control of many LEDs is essential for a successful market entry. Figure 1 depicts an intelligent lighting control system that enables such intuitive control. The technology presented in this paper is believed to enable new easy-to-use concepts for control of a large number of LEDs in a lighting environment. This technology is based on modulation of the light emitted by LEDs (via "light link c") and a sensor receiving the information transmitted by the LEDs and estimating the light intensity of each LED. In an intelligent lighting system, these estimates would be, together with user input on required lighting pattern, transmitted to a master controller (via "control link a") connected to all the LED lamps (via "control link b"). The data transmitted over "link a" is generally low-rate individual data of the lamp, such as type number, burning hours or lamp temperature. In a typical system "link a" would be a radio frequency (RF) link and "link b" either a wired or RF wireless link. In this paper we investigate the visible light link c.

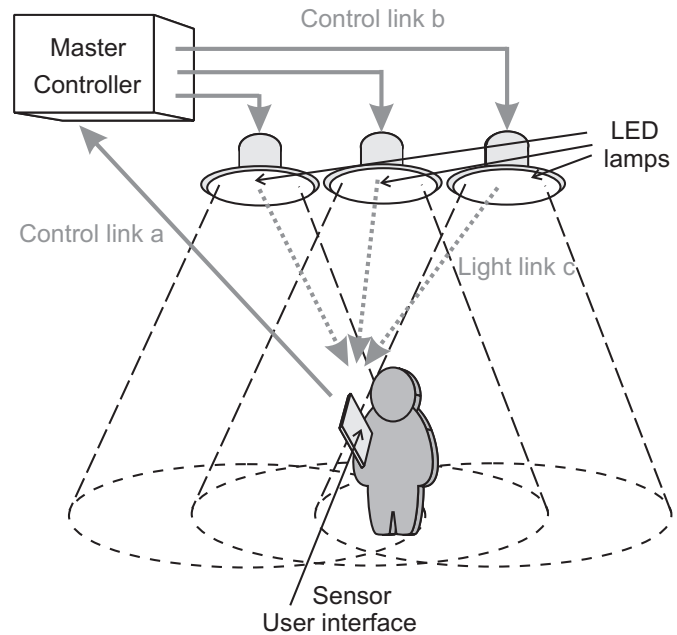

Fig. 1. An illustration of an intelligent lighting control system.

Modulation of LEDs has been studied in several papers, though initially mainly for infrared (IR) communications [2], [3], [4]. Currently we observe an increased interest in visible light communications using powerful lighting LEDs, e.g. [5], [6]. A few papers further address the combination of pulsewidth dimming with data modulation, e.g. to carry a common signal on all LEDs. Recently, code division multiple access (CDMA) for IR communications has been treated in [7].

We propose a modulation and multiple-access scheme, called CTDMA-PPM: a form of pulse-position modulation which is keyed according to a spreading sequence, and in which the duty cycle is subject to PWM according to the required dimming levels. To our knowledge our current paper is the first in specifically addressing CDMA "coded light" for the application of lighting control, simultaneously addressing the communication and lighting constraints, e.g. bit-error rate and dimming level, respectively. Moreover, what we propose also differs from the modulation method proposed as PPMCDMA in [8], which time-shifts an entire pre-defined CDMA code sequence according to the user data bits. In contrast to PPM-CDMA, in our CTDMA-PPM scheme, the exclusive OR of the user data and the code sequence determines the individual position of each pulse within a series. As a consequence, our waveforms for representing the information bit " 0 " and "1" are not time-shifted copies of each other, as in [8]. This allows us to distinguish LEDs not only based by their code but also by their time offset, hence we exploit a hybrid code-time division multiple access (CTDMA). To obtain a pronounceable acronym, we nicknamed the system LED-NET. 
This paper is organized as follows. First, Section II formulates the LED-NET encoding system. Subsequently, Section III describes the channel, and Section IV presents a receiver structure. The performance of the proposed system is illustrated with numerical results in Section V. Finally, Section VI concludes this paper.

\section{Modulation And Multiple Access}

In this section, we propose the CTDMA-PPM visible light modulation scheme. We propose a three-layer structure and use the following nomenclature for modulated pulses:

- A slot has duration $T_{1}$. It represents the clock timing at which the high brightness LEDs are driven. Hence $T_{1}$ is the resolution at which the LEDs can be modulated.

- A block has duration $T_{2}=N_{1} T_{1}$ and represents the duration of one code chip. The ratio $1: N_{1}$ is the contrast ratio and also the resolution of the possible adjustment of the duty cycle for illumination purposes. This corresponds to $q=\log _{2} N_{1}$ bits in dimming capabilities. In one block duration an LED emits one pulse. The unique property of this modulation method is that the pulse can extend beyond the block boundary, according to the illumination settings.

- A frame has duration $T_{3}=N_{2} T_{2}$ and is the time interval during which one information bit is send, and one measurement of the illumination intensity of each LED is made.

\section{A. Block Modulation Format: Pulse Position Modulation}

Here we introduce binary PPM for a pulse of amplitude $A_{l}$ and timing reference $\tau_{l}$ with $\tau_{l} \in\left\{0,1, \ldots, N_{1}-1\right\}$.

- The starting position of the $j$ th pulse for the $l$ th LED, with $j=0, \ldots, N_{2}-1$ and $l=1, \ldots, L$, is given by

$$
\delta_{j, l}= \begin{cases}\tau_{l}, & a_{j, l}=-1 \\ \tau_{l}+k_{l}, & a_{j, l}=1\end{cases}
$$

where $\tau_{l}$ and $k_{l}$ are the allocated time slot and the modulation depth for the $l$ th LED, respectively, and

$$
a_{j, l}=b_{l} c_{j, l},
$$

where $b_{l} \in\{+1,-1\}$ is the information bit from the $l$ th LED. Here $c_{j, l}$ denotes the $j$ th chip of its spreading sequence with $c_{j, l} \in\{+1,-1\}$. The length of the spreading sequence is $N_{2}$.

- The pulse width in terms of number of slots is given by $w_{l}=\operatorname{round}\left(p_{l} N_{1}\right)$, where $p_{l}$ is the dimming level of the $l$ th LED, with $0 \leq p_{l} \leq 1$.

Figure 2 illustrates a binary PPM pulse, as described above.

Evidently, if we need to communicate data, we cannot switch off the light completely. In PPM, light is emitted in at least one slot, so the realized $\hat{p}_{l} \geq 1 / N_{1}$. If the total light level in a room must be dimmable to non-perceivable illumination levels this may represent a problem. On the other hand, the minimum light level can be made arbitrarily small by choosing appropriate $T_{1}$ and $A_{l}$. The fact that the duty cycle also needs to be slightly below $100 \%$ does not lead to any practical limitation because it has only a minor impact on the output light intensity of an LED.

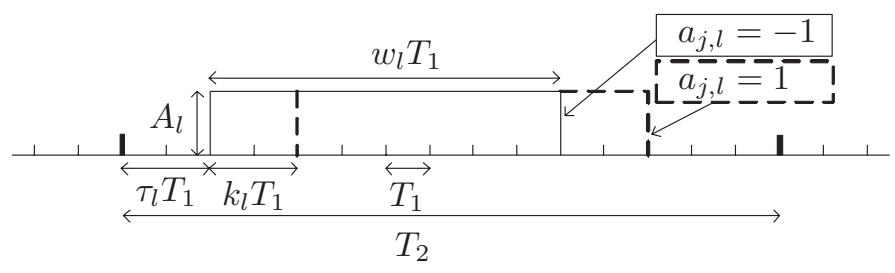

Fig. 2. Description of the binary PPM modulation format.

\section{B. Framing}

We address a synchronous system in which $T_{1}, N_{1}$ and $N_{2}$ are identical for all LEDs, and their slots, blocks and frames are aligned. We will omit the notational details of pulses extending into neighboring blocks, and we only consider the first frame in a continuous sequence of frames. The driving signal for the $l$ th LED can be expressed as

$$
s_{l}(t)=\sum_{j=0}^{N_{2}-1} \sum_{n=0}^{N_{1}-1} s_{j, n, l} \Pi\left(\frac{t-j T_{2}-n T_{1}}{T_{1}}\right) .
$$

Here $n$ and $j$ refer to the positions of slots in a block, and to blocks in the frame, respectively, and $\Pi$ is the unit pulse of unit width and amplitude. The discrete-time signal $s_{j, n, l}$ represents the sample of $s_{l}(t)$ at $t=j T_{2}+n T_{1}$. For PPM it is given by

$$
s_{j, n, l}=A_{l} \cdot \begin{cases}0 & n=0,1, \ldots, \tau_{l}-1 \\ \frac{1-a_{j, l}}{2} & n=\tau_{l}, \ldots, \tau_{l}+k_{l}-1 \\ 1 & n=\tau_{l}+k_{l}, \ldots, \tau_{l}+w_{l}-1 \\ \frac{1+a_{j, l}}{2} & n=\tau_{l}+w_{l}, \ldots, \tau_{l}+w_{l}+k_{l}-1, \\ 0 & n=\tau_{l}+w_{l}+k_{l}, \ldots, N_{1}-1 .\end{cases}
$$

\section{Multiple Access}

CDMA allows multiple light sources to simultaneously emit PWM light and communicate data. Walsh-Hadamard (WH) codes can ensure perfect orthogonality and allow computationally efficient multi-signal receiver algorithms. By excluding the first WH code, namely the $\{1,1,1, \ldots\}$ DC word, all codes used have a balanced number of 1's and -1 's. This further shapes the illumination spectrum. Moreover, the system becomes resilient to sources of constant or sufficiently slowly varying interfering light sources such as sunlight or incandescent bulbs. The $l$ th LED is assigned a spreading sequence indexed by $\gamma_{l}$.

We, additionally, assign a timing reference $\tau_{l}$ to each LED, where each combination $\left(\tau_{l}, \gamma_{l}\right)$ is unique in the system, i.e., characterizes an LED. The upper bound to the number of orthogonal LEDs thus equals $N_{1} N_{2}$. Since the data is contained in the start and end of the pulse (see Fig. 2 and (4)), however, it is more convenient to limit the number of LEDs to $N_{1} N_{2} / 2$, which allows for a lower receiver complexity.

For CTDMA-PPM, code and time reference assignment can be challenging because of the arbitrary and possibly varying illumination pulse duration. Hence, a pragmatic approach is to let $\tau_{l}$ be the same for every LED and only assign a unique code $\gamma_{l}$. 


\section{Channel Model}

The electrical channel is described by the relation between the signal $s_{l}(t)$ output of the $l$ th LED driver (the transmitter) and signal $y(t)$ that is offered to the receiver. The corresponding optical transmit and receive signals are denoted as $\varsigma_{l}(t)$ and $\chi(t)$, respectively.

\section{A. Electro-Optical Conversion}

The optical transmit signal $\varsigma_{l}(t)$ in response to a step function of the electrical transmit signal $s_{l}(t)=A_{l} U(t)$ is given by $\varsigma_{l}(t)=A_{l} \eta_{l} h_{\mathrm{on}}(t)$ [9], where $\eta_{l}$ denotes the LED responsivity. The unit step response of LEDs appears approximately an exponential function [10], i.e.,

$$
\begin{aligned}
& h_{\mathrm{on}}(t)=U(t)\left[1-\exp \left(-t / \tau_{\text {on }}\right)\right], \\
& h_{\text {off }}(t)=1-U(t)\left[1-\exp \left(-t / \tau_{\text {off }}\right)\right] .
\end{aligned}
$$

with $U(t)$ the unit step function. The time constants $\tau_{\text {on }}$ and $\tau_{\text {off }}$ for on- and off-switching tend to differ [10]. These switching effects are considered here, since they are much more pronounced in power and phosphor-coated LEDs than in typical LEDs designed for IR communications. Ignoring a possibly more complicated interaction between the on- and off-tails for very short pulses, the CTDMA-PPM optical signal becomes

$$
\begin{aligned}
\varsigma_{l}(t)=\sum_{j=0}^{N_{2}-1} A_{l} \eta_{l} h_{\mathrm{on}}\left(t-\delta_{j, l} T_{1}-j T_{2}\right) \\
\cdot h_{\mathrm{off}}\left(t-\left(\delta_{j, l}+w_{l}\right) T_{1}-j T_{2}\right) .
\end{aligned}
$$

\section{B. Indoor Light Propagation}

The LED light reaches the sensor possibly via multiple paths, as studied for instance by [2], [3]. However, we consider LEDs with narrow beams in this work, since we want to enable illumination pattern rendering. Consequently, reflected light from these LEDs reaching the sensor has experienced multiple reflections and will be largely attenuated. Hence, for the analysis in this paper, we focus on the line-of-sight path. The gain of such an optical path is

$$
\alpha_{l}=\frac{1}{r_{l}^{2}} R\left(\phi_{l}\right) \mathcal{A} \cos \left(\psi_{l}\right),
$$

where $r_{l}$ is the propagation distance, $\mathcal{A}$ is the area of the photodiode (PD) used as part of the sensor and the angle $\psi_{l}$ defines the orientation of LED $l$. Further, $R\left(\phi_{l}\right)$ describes the LED radiation pattern, which we assume to be rotationally symmetric and includes the effects of the lens. Further, the angle $\phi_{l}$ defines the orientation of the PD with respect to the $l$ th LED. We can model $R\left(\phi_{l}\right)$ by a generalized Lambertian law [9], [2]

$$
R\left(\phi_{l}\right)=\frac{\mu+1}{2 \pi} \cos ^{\mu}\left(\phi_{l}\right),
$$

where $\mu$ represents the Lambertian mode number.

\section{Opto-Electrical Conversion}

The PD in the receiver converts the optical signal $\chi(t)$ into the electrical signal $y(t)$. Its responsivity is $\varepsilon$. The speed of PDs is generally much higher than that of power LEDs, hence, switching effects can be considered negligible [9], [11].

\section{Channel Disturbances}

There are two major sources of channel disturbances, i.e. the electronics noise and shot noise. Electronics noise is mostly created by the transimpedance amplifier for the PD signal, and predominantly behaves as additive Gaussian (thermal) noise. For a power spectral density (PSD) $S_{\mathrm{th}}\left[\mathrm{A}^{2} / \mathrm{Hz}\right]$ in an effective bandwidth $B_{\mathrm{n}}=1 / T_{1}$, the variance of the thermal noise equals

$$
\sigma_{\mathrm{th}}^{2}=S_{\mathrm{th}} B_{\mathrm{n}} .
$$

Shot noise is due to a stream of electrons that are generated at random times in the PD. It is approximately Gaussian distributed due to the central limit theorem [3]. The shot noise power $\sigma_{\text {shot }}^{2}$ is linearly proportional to all light shed on the PD surface, not only including the light from LEDs, but also the background light. Hence it can be expressed as

$$
\sigma_{\text {shot }}^{2}(t)=2 q_{\mathrm{e}} B_{\mathrm{n}} \varepsilon\left(\zeta+\sum_{l=1}^{L} \alpha_{l} \varsigma_{l}(t)\right),
$$

where $q_{\mathrm{e}}$ is the electrical charge of an electron $\left(q_{\mathrm{e}}=1.6 \cdot 10^{-19}\right.$ Coulomb), $\zeta$ is the background light power, which can be assumed to be approximately DC, and $L$ is the number of LEDs. As an approximation, we assume that the sum of all the light is time independent, e.g., due to strong background light. Hence, the argument $t$ of $\sigma_{\text {shot }}^{2}$ will be dropped in the following sections.

Hence, the received electrical signal is given by

$$
y(t)=\sum_{l=1}^{L} \varepsilon \alpha_{l} \varepsilon \varsigma_{l}(t)+v_{\zeta}+v_{\text {th }}(t)+v_{\text {shot }}(t),
$$

where $v_{\zeta}=\varepsilon \zeta, v_{\text {th }}(t)$ and $v_{\text {shot }}(t)$ denote the background light contribution, thermal and shot noise terms, respectively. The variances for the latter two are defined in (10) and (11), respectively.

\section{Receiver Design and Performance Analysis}

In the receiver, we first apply integrate-and-dump (I\&D) processing, followed by CDMA despreading to obtain the despreaded signal values for each $\tau$. The I\&D receiver outputs the $N_{2} \times N_{1}$ matrix $\mathbf{Y}$, whose $(j, n)$ th element equals

$$
y_{j, n}=\frac{1}{T_{1}} \int_{t=j T_{2}+(n-1) T_{1}}^{t=j T_{2}+n T_{1}} y(t) \mathrm{d} t .
$$

For CDMA despreading, we use a $N_{2} \times 2 k_{l}$ submatrix of $\mathbf{Y}$, denoted by $\mathbf{Y}_{l}$, containing the columns $n=\tau_{l}, \ldots, \tau_{l}+k_{l}-1$ and $n=\tau_{l}+w_{l}, \ldots, \tau_{l}+w_{l}+k_{l}-1$ of $Y$, which are the data measurements related to the lth LED. The despreaded signal can be collected in the $2 k_{l} \times 1$ vector

$$
\mathbf{d}_{l}=\frac{2}{N_{2}} \mathbf{Y}_{l}^{T} \mathbf{c}_{l}=g_{l} b_{l} \mathbf{h}_{l}+\mathbf{d}_{\text {noise }},
$$

where $\mathbf{c}_{l}=\left[c_{1, l}, c_{2, l}, \cdots c_{N_{2}, l}\right]^{T}$ is the Walsh-Hadamard codeword corresponding to the $l$ th LED, $\mathbf{d}_{\text {noise }}$ is the noise component, including both the thermal and shot noises, and its variance is $\sigma^{2}=4\left(\sigma_{\text {th }}^{2}+\sigma_{\text {shot }}^{2}\right) / N_{2}$. The individual light intensity from the $l$ th LED at the sensor location is given by $g_{l}=$ $A_{l} \alpha_{l} \eta_{l}$. The $2 k_{l} \times 1$ vector $\mathbf{h}_{l}$ is known to the receiver and can be written as $\mathbf{h}_{l}=\varepsilon\left[h_{1, \text { on }}, \cdots, h_{k_{l}, \text { on }}, h_{1, \text { off }}, \cdots, h_{k_{l}, \text { off }}\right]^{T}$, if 
$\tau_{\text {on }}$ and $\tau_{\text {off }}$ are small with respect to $w_{l}$. Here, due to the I\&D receiver structure, we get

$$
\begin{aligned}
h_{i, \text { on }} & =\frac{1}{T_{1}} \int_{(i-1) T_{1}}^{i T_{1}}\left[1-\exp \left(-t / \tau_{\text {on }}\right)\right] \mathrm{d} t \\
& =1-\frac{\tau_{\text {on }}}{T_{1}}\left[\exp \left(-(i-1) T_{1} / \tau_{\text {on }}\right)-\exp \left(-i T_{1} / \tau_{\text {on }}\right)\right],(15)
\end{aligned}
$$

and similarly,

$h_{i, \text { off }}=1-\frac{\tau_{\text {off }}}{T_{1}}\left[\exp \left(-(i-1) T_{1} / \tau_{\text {off }}\right)-\exp \left(-i T_{1} / \tau_{\text {off }}\right)\right]$.

\section{A. Bit Detection}

For bit detection, the elements of $\mathbf{d}_{l}$ are combined through maximum ratio combining (MRC) followed by a threshold detector. The bit-error rate (BER) for the $l$ th LED equals

$$
\begin{aligned}
\mathrm{BER}_{l} & =\frac{1}{2} \operatorname{erfc}\left(\sqrt{\mathrm{SNR}_{l} / 2}\right) \\
& =\frac{1}{2} \operatorname{erfc}\left(\sqrt{\frac{N_{2} g_{l}^{2} \varepsilon^{2} \sum_{i=1}^{k_{l}}\left(h_{i, \text { on }}^{2}+h_{i, \text { off }}^{2}\right)}{8\left(\sigma_{\text {th }}^{2}+\sigma_{\text {shot }}^{2}\right)}}\right)
\end{aligned}
$$

where $\operatorname{erfc}(\cdot)$ is the complementary error function. The lower bound of the BER is achieved when $\tau_{\text {on }}=\tau_{\text {off }}=0$ and is given by

$$
\mathrm{BER}_{l} \geq \frac{1}{2} \operatorname{erfc}\left(\sqrt{\frac{N_{2} k_{l} g_{l}^{2} \varepsilon^{2}}{4\left(\sigma_{\text {th }}^{2}+\sigma_{\text {shot }}^{2}\right)}}\right) .
$$

It can be concluded from the above, that due to the effect of on- and off-switching ramps, or the effect of large $\tau_{\text {on }}$ and $\tau_{\text {off }}$, the BER performance suffers from loss in SNR compared to the lower bound. It can also be concluded that an increase in code length $N_{2}$ and modulation depth $k_{l}$ increases the BER performance.

\section{B. Intensity Estimation}

It was explained in Section I that it is essential to measure the individual light level $g_{l}$ in an intelligent lighting system. A linear estimator of $\hat{g}_{l}$ can be written as

$$
\hat{g}_{l}=\boldsymbol{\xi}_{l}^{T} \mathbf{d}_{l} .
$$

One can choose the weight vector $\boldsymbol{\xi}_{l}$ according to the least squares (LS) and the minimum mean square error (MMSE) criteria, which reflect the cases that the variance of $\mathbf{d}_{\text {noise }}$ is unknown and known, respectively.

1) LS Estimation: For LS estimation of $g_{l}$, the weight vector becomes

$$
\boldsymbol{\xi}_{l, \mathrm{LS}}=\left(\hat{b}_{l} \mathbf{h}_{l}^{T} \mathbf{h}_{l}\right)^{-1} \mathbf{h}_{l}=\frac{\hat{b}_{l}^{-1} \mathbf{h}_{l}}{\varepsilon^{2} \sum_{i=1}^{k_{l}}\left(h_{i, \mathrm{on}}^{2}+h_{i, \mathrm{off}}^{2}\right)},
$$

where $\hat{b}_{l}$ represents the received bit. For performance analysis, we use

$$
\hat{g}_{l, \mathrm{LS}}=g_{l}+\left(\hat{b}_{l} \mathbf{h}_{l}^{T} \mathbf{h}_{l}\right)^{-1} \mathbf{h}_{l}^{T} \mathbf{d}_{\text {noise }}
$$

The second term is the least squares error (LSE), which is a zero-mean random Gaussian variable with mean squared value

$$
\sigma_{\text {LSE }}^{2}=\left(\mathbf{h}_{l}^{T} \mathbf{h}_{l}\right)^{-2} \mathbf{h}_{l}^{T} \mathbf{R}_{\text {noise }} \mathbf{h}_{l},
$$

where $\mathbf{R}_{\text {noise }}=\mathbb{E}\left[\mathbf{d}_{\text {noise }} \mathbf{d}_{\text {noise }}^{T}\right]$. If the noise is i.i.d, i.e. $\mathbf{R}_{\text {noise }}=$ $\sigma^{2} \mathbf{I}$, where $\mathbf{I}$ is the identity matrix, then

$$
\sigma_{\mathrm{LSE}}^{2}=\frac{4\left(\sigma_{\mathrm{th}}^{2}+\sigma_{\text {shot }}^{2}\right)}{N_{2} \varepsilon^{2} \sum_{i=1}^{k_{l}}\left(h_{i, \text { on }}^{2}+h_{i, \text { off }}^{2}\right)} .
$$

Further, similarly as shown in the BER performance, the lower bound of LSE is given by

$$
\sigma_{\text {LSE }}^{2} \geq 2\left(\sigma_{\text {th }}^{2}+\sigma_{\text {shot }}^{2}\right) /\left(k_{l} N_{2} \varepsilon^{2}\right) .
$$

Similar to the bit detection, the LS estimation process also suffers from a reduction of SNR due to the non-ideal response of the LEDs.

2) Linear MMSE Estimation: The LS intensity estimator is unbiased, but suffers from noise enhancement. MMSE estimation for known $\mathbf{R}_{\text {noise }}$ can be realized by estimating the variance of the shot noise through the DC-code contribution.

The MMSE estimator can be shown to be given by

$$
\boldsymbol{\xi}_{l, \mathrm{MMSE}}=\hat{b}_{l}\left(1 / \mathbb{E}\left[g_{l}^{2}\right]+\mathbf{h}_{l}^{T} \mathbf{R}_{\text {noise }}^{-1} \mathbf{h}_{l}\right)^{-1} \mathbf{R}_{\text {noise }}^{-1} \mathbf{h}_{l},
$$

and the resulting mean squared estimation error equals

$$
\sigma_{\text {MMSE }}^{2}=\left(1 / \mathbb{E}\left[g_{l}^{2}\right]+\mathbf{h}_{l}^{T} \mathbf{R}_{\text {noise }}^{-1} \mathbf{h}_{l}\right)^{-1} .
$$

Similarly as for the LS estimator, the lower bound of $\sigma_{\text {MMSE }}^{2}$ can be obtained as

$$
\sigma_{\mathrm{MMSE}}^{2} \geq \frac{\sigma_{\mathrm{th}}^{2}+\sigma_{\mathrm{shot}}^{2}}{\frac{\sigma_{\mathrm{th}}^{2}+\sigma_{\text {shot }}^{2}}{\mathbb{E}\left[g_{l}^{2}\right]}+\frac{k_{l} N_{2} \varepsilon^{2}}{2}} .
$$

Hence, when the signal-to-noise ratio is high, the MMSE estimator and LS estimator tend to become equivalent.

\section{Numerical Results}

In this section, we present the numerical results calculated based on a scenario for a typical large indoor environment. In this scenario, illumination LEDs are distributed uniformly over the ceiling, with the density of $30 \mathrm{LEDs} / \mathrm{m}^{2}$. The Lambertian mode number $\mu$ is the same for all LEDs. The output light power of each LED is 0.5 Watt. Walsh-Hadamard codes with $N_{2}=256$ are used, and $N_{1}$ is set to be 1024 to provide a dimming range of 10 bits. Every LED takes $k=4$ slots for modulation. The slot period $T_{1}=1 \mu \mathrm{s}$. We assume that $\tau_{\text {on }}<<T_{1}$ and $\tau_{\text {off }}<<T_{1}$ and that the source of noise includes electronics and shot noises. The sensor is oriented such that it faces the ceiling at the distance of $3 \mathrm{~m}$. Other system parameters are listed in Table I. While all the LEDs are radiating light and transmitting data simultaneously, we consider only the communications and sensing performances of the link between one LED and the sensor.

In Fig. 3, we present BER results as a function of $r_{l}$, the propagation distance between the LEDs and the sensor, for LEDs with different Lambertian mode numbers $\mu$. The sensor is located three meters below the ceiling and we take numerical 
TABLE I

Simulation PARAmeters

\begin{tabular}{|l|l|}
\hline parameters & values \\
\hline Background light power & $5 \mathrm{~W} / \mathrm{m}^{2}$ \\
\hline Distance between sensor and ceiling & $3 \mathrm{~m}$ \\
\hline Duty cycle of each LED, $p_{l}$ & $50 \%$ \\
\hline Responsivity of photodiode, $\varepsilon$ & 0.3217 \\
\hline PSD of electronics noise, $S_{\mathrm{th}}$ & $1.69 \times 10^{-24}$ \\
\hline area of the PD, $\mathcal{A}$ & $10^{-4} \mathrm{~m}^{2}$ \\
\hline
\end{tabular}

results for LEDs at different locations on the ceiling. We can observe that the communication link is almost errorless up to a range of 10,8 and $6 \mathrm{~m}$, for $\mu$ equals 1,2 and 5 , respectively. Beyond this range, the BER increases dramatically as the sensor moves away from the center of the LED light beam and as a consequence of the increase in free space loss.

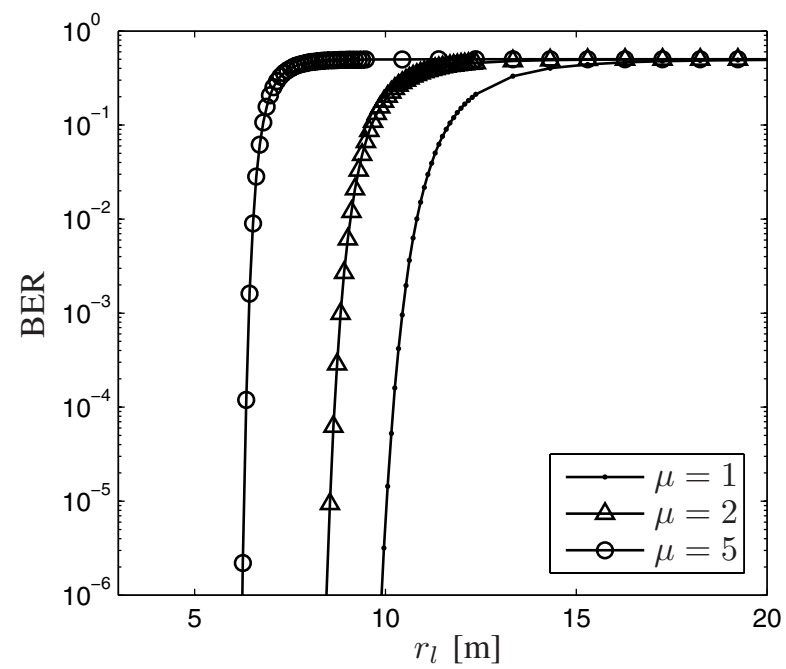

Fig. 3. BER vs. PD-LED distance.

Figure 4 depicts the normalized MSE (N-MSE) in light intensity estimation as a function of distance, for LEDs with different $\mu$. The N-MSE is defined as the MSE normalized by the light intensity. It is shown in Fig. 4 that the performance in intensity estimation degrades in an exponential fashion as the distance between the LED and sensor increases. We can observe that we get considerable estimation errors, $\mathrm{N}$-MSE $>10^{-2}$, in the intensity estimation for LEDs whose distances from the sensor are above 12, 10 and 7 meters for $\mu$ equals 1,2 and 5, respectively. The impact of this error, however, is limited since the contribution to the local illumination from LEDs which are beyond these distances is negligible, i.e. their contributions are more than $20 \mathrm{~dB}$ lower than that of the contribution of the locally dominant LED.

\section{Discussions ANd Conclusions}

In this paper, we showed an approach to simultaneously illuminate and transmit information from individual illumination LEDs. In order to create desired lighting patterns, it is essential to identify and estimate the light contribution of each LED in a localized manner. To this end, a new light modulation method and a multiple access scheme is proposed,

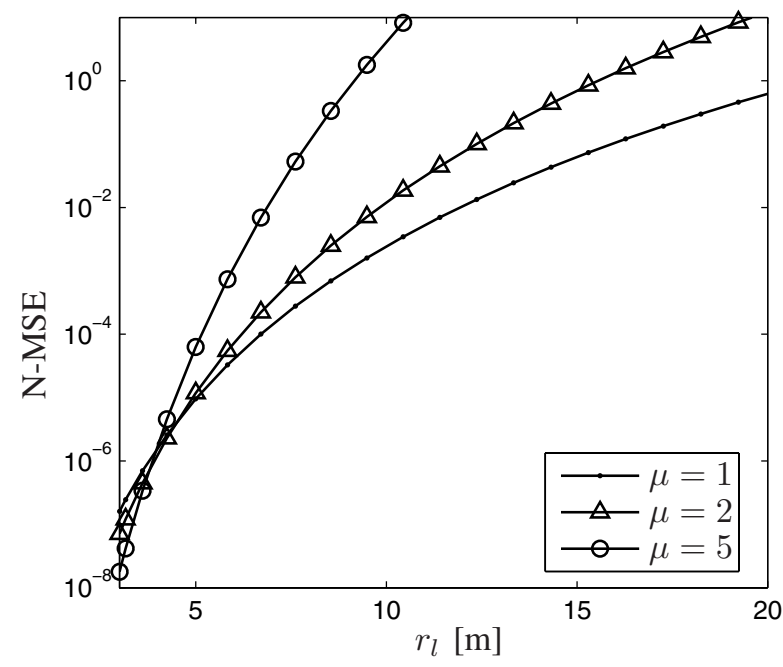

Fig. 4. Normalized MSE in intensity estimation vs. PD-LED distance.

combined with illumination dimming capabilities, for the emerging illumination LED technology. It is named code-time division multiple access - pulse position modulation (CTDMAPPM). Further research can be done to define constraints on the PPM pulses to ensure full orthogonality. To estimate the light contributions of each LED at the sensor location we studied the least squares and minimum mean square error estimators. The communication performance if found by the derivation of the BER model.

We conclude that for practical parameters in a realistic scenario, our approach provides the required performance up to the range of about 10 meters, with a large number of LEDs, a small detector, and under a strong background light.

\section{ACKNOWLEDGMENTS}

The authors thank prof. Jan Bergmans, prof. Shlomi Arnon, dr. Stan Baggen and dr. Joop Talstra for their valuable input.

\section{REFERENCES}

[1] E. F. Schubert and J. K. Kim, "Solid-state light sources getting smart," Science, vol. 308, pp. 1274-1278, May 2005.

[2] F. R. Gfeller and U. Bapst, "Wireless in-house communication via diffuse infrared radiation," Proc. IEEE, vol. 67, no. 11, pp. 1474-1486, Nov. 1979.

[3] J. M. Kahn and J. R. Barry, "Wireless infrared communications," Proc. IEEE, vol. 85, no. 2, pp. 265-298, Feb. 1997.

[4] R. Otte, L. P. de Jong, and A. H. M. van Roermund, Low-power Wireless Infrared Communications. Springer, 1999.

[5] T. Komine and M. Nakagawa, "Fundamental analysis for visible-light communication system using LED lights," IEEE Trans. Consumer Electron., vol. 50, no. 1, pp. 100-107, Feb. 2004.

[6] G. Pang, T. Kwan, H. Liu, and C.-H. Chan, "LED wireless," IEEE Ind. Appl. Mag., vol. 8, no. 1, pp. 21-28, Jan./Feb. 2002

[7] U. N. Griner and S. Arnon, "Multiuser diffuse indoor wireless infrared communications using equalized synchronous CDMA," IEEE Trans. Commun., vol. 54, pp. 1654-1662, Sept. 2006.

[8] J. M. H. Elmirghani and R. A. Cryan, "New PPM-CDMA hybrid for indoor diffuse infrared channels," Electronics Letters, vol. 30, pp. 16461647, September 1994.

[9] D. Wood, Optoelectronic Semiconductor Devices. Prentice Hall, 1994.

[10] A. Descombes and W. Guggenbuhl, "Large signal circuit model for LED's used in optical communication," IEEE Trans. Electron Devices, vol. ED-28, no. 4, pp. 395-404, Apr. 1981

[11] A. E. Iverson and D. L. Smith, "Mathematical modeling of photoconductor transient response," IEEE Trans. Electron Devices, vol. 34, pp. 2098-2107, Oct. 1987. 